\title{
Optical Communication Using Subcarrier PSK Intensity Modulation Through Atmospheric Turbulence Channels
}

\author{
Jia Li, Member, IEEE, John Q. Liu, Senior Member, IEEE, and Desmond P. Taylor, Life Fellow, IEEE
}

\begin{abstract}
This paper studies optical communications using subcarrier phase shift keying (PSK) intensity modulation through atmospheric turbulence channels. The bit error rate (BER) is derived for optical communication systems employing either on/off key (OOK) or subcarrier PSK intensity modulation. It is shown that at $\mathrm{BER}=10^{-6}$ and a scintillation level of $\sigma=0.1$, an optical communication system employing subcarrier BPSK is $3 \mathrm{~dB}$ better than a comparable system using fixed-threshold OOK. When $\sigma=0.2$, an optical communication system employing subcarrier BPSK achieves a $\mathrm{BER}=10^{-6}$ at $\mathrm{SNR}=13.7 \mathrm{~dB}$, while the $\mathrm{BER}$ of a comparable system employing OOK can never be less than $10^{-4}$. Convolutional codes are discussed for optical communication through atmospheric turbulence channels. Interleaving is employed to overcome memory effect in atmospheric turbulence channels. An upper bound on BER is derived for optical communication systems employing convolutional codes and subcarrier BPSK modulation.
\end{abstract} tion.

Index Terms-Atmospheric turbulence, coding, laser, scintilla-

\section{INTRODUCTION}

$\mathbf{O}$ PTICAL communication technology has been shown to be a good wireless interconnect technology for highcapacity communication networks in the first and last mile [1]-[3]. A big challenge in optical wireless communications is to mitigate signal scintillation introduced by atmospheric turbulence. Turbulence is caused by inhomogeneities of both temperature and pressure in the atmosphere [4]-[6], and is responsible for the refractive index variation of the air. Turbulence causes amplitude and phase fluctuations in the received optical beam. Such fluctuations deteriorate signal intensity at the receiver, increase bit error rate (BER), and can break the communication link [5]. Field measurements were performed in [5] with a link of $2.4 \mathrm{~km}$ in San Diego on February 25, 1997, a sunny and warm day. It was found that the scintillation was on the order of $2-4 \mathrm{~dB}$

Paper approved by J. A. Salehi, the Editor for Optical CDMA of the IEEE Communications Society. Manuscript received August 26, 2005; revised February 23,2006, and June 12, 2006. This work was supported in part by the National Science Foundation under Grant CNS-0435341 and Grant CNS-0702874, and in part by the U.S. Army Research Office (ARO) under Grant W911NF0410267. This paper was presented in part at the IEEE Global Telecommunication Conference, Dallas, TX, November 29-December 3, 2004, and at the IEEE International Conference on Communications, Seoul, Korea, May 16-20, 2005.

$\mathrm{J}$. Li is with the Department of Electrical and Computer Engineering, Oakland University, Rochester, MI 48309 USA (e-mail: li4@oakland.edu).

J. Q. Liu is with the Department of Electrical and Computer Engineering, Wayne State University, Detroit, MI 48202 USA (e-mail: jliu@eng.wayne.edu).

D. P. Taylor is with the Electrical and Computer Engineering Department, University of Canterbury, Christchurch 8140, New Zealand (e-mail: taylor@elec.canterbury.ac.nz).

Digital Object Identifier 10.1109/TCOMM.2007.902592 at 7:10 Pacific Standard Time (PST) and 17:30 PST, and reached $29 \mathrm{~dB}$ at 13:20 PST. The scintillation was worst around 1:30 pm, and was very bad for several hours [5]. Signal scintillation caused by atmospheric turbulence severely degrades the performance of optical communication systems [7], [8]. It severely limits the applications of optical wireless communications [9].

The effect of atmospheric turbulence on light waves was extensively studied in the 1960s [8]. The scintillation introduced by turbulence was studied in [10] through experiments. It was shown that there was a limit for aperture averaging to mitigate optical signal scintillation. When the detector collection aperture reaches some certain size, increasing it will not help further reduce the scintillation level [10, Fig. 4]. Measurements were made in [11] with collection aperture ranging from $1 \mathrm{~mm}$ to $1 \mathrm{~m}$ in diameter. The log-normal variance decreased smoothly for diameters from $1 \mathrm{~mm}$ to about $10 \mathrm{~cm}$, and showed no decrease for receiver aperture from $10 \mathrm{~cm}$ to $1 \mathrm{~m}$. Theory and experimental results on aperture averaging were discussed in [12]. The receiver aperture in [13] was $D=8 \mathrm{~cm}$, much larger than the correlation length of $d_{0}=1.8 \mathrm{~cm}$ for a $500 \mathrm{~m}$ outdoor link. The weak turbulence in [13] degraded SNR by $16 \mathrm{~dB}$ at a $\mathrm{BER}=2 \times 10^{-4}$. Experiments showed that the signal scintillation magnitude saturates at a range of about $700 \mathrm{~m}$ [14], [15]. No further growth of scintillation magnitude was observed at longer distance. In these experiments, the magnitude of scintillation was observed as a log-normal distribution. A theoretical approximation was developed in [16] to show that optical signal scintillation caused by strong atmospheric turbulence has a log-normal distribution.

Recently, progresses have been made to mitigate optical signal scintillation caused by atmospheric turbulence. Spatial diversity was employed in [17], where multiple copies of a message were sent through uncorrelated paths to a receiver. Multiple copies of the received signal were combined at the receiver to make a decision. Such an optical communication system employs $N>1$ transmitting optical beams and $M \geq 1$ front end(s) in the receiver [18]. Terminal cost increases with $N$ and $M$. Adaptive optics to mitigate optical signal scintillation was analyzed in [19]. Adaptive optics needs complicated design, and results in high cost.

Communication theory to overcome optical signal scintillation caused by atmospheric turbulence is far from being mature. The existing work related to high data rate commercial applications has focused on communication systems using OOK modulation [9], [13], [18], [20], [21]. In such a system, a threshold is set for demodulation. Atmospheric turbulence makes it 
very difficult to choose an appropriate threshold [7]. Thousands of optical wireless communications terminals deployed in the past decade have employed OOK with fixed threshold, for its simplicity in implementation. However, atmospheric turbulence remains as a big problem for OOK systems [7], [13], [18]. Existing systems have employed high transmission power to handle atmospheric turbulence. This practice has resulted in high cost.

Subcarrier BPSK intensity modulation in optical wireless communications was briefly analyzed in [22]. An experiment on differential BPSK was reported. It was shown that subcarrier phase-shift keying (PSK) intensity modulation was superior to OOK in the presence of atmospheric turbulence. The OOK curves in [22] were directly from [7]. No solid analysis has been developed to answer why subcarrier PSK intensity modulation can perform better. Because the optical wireless communications industry has experienced a healthy growth in recent years, it is important to develop theory and technology to overcome atmospheric turbulence and reduce system cost. There is a need to compare OOK against subcarrier PSK intensity modulation through solid analysis in modulation theory. Such an analysis will clear doubts on optical wireless communications, and should support the design of future optical wireless communications systems. Performance of coded systems employing subcarrier PSK intensity modulation also needs to be investigated for the selection of error correcting codes in practice.

This paper develops a framework for optical communications through a turbulent atmosphere. Section II describes the system model. Section III analyzes BER for optical communication systems using OOK. Its intrinsic disadvantage is identified. Section IV analyzes the BER for optical communication systems employing subcarrier PSK intensity modulation. Section V employs interleaving to improve decoder performance and simplify the analysis of BER bound for convolutional codes. Simulation results are presented for optical communications systems employing convolutional codes with either OOK or subcarrier PSK intensity modulation. Section VI concludes the study.

\section{SYSTEM MODEL}

Fig. 1 shows the block diagram of an optical communication system through the atmosphere. The information generated by a source is encoded by an encoder, interleaved, and modulated into an electrical waveform by an electrical modulator. In the optical modulator, the intensity of a light source is modulated by the output signal of the electrical modulator. The light source is a laser, characterized by its wavelength, power, and beam divergence angle. There is a collimator or telescope in the transmitter to determine the direction and the size of the laser beam. The receiver consists of an optical front end, a photodetector, a demodulator, a deinterleaver, and a decoder. The optical front end contains lenses focusing the received optical field onto a photodetector. The photodetector converts the received optical field to an electronic signal, which is demodulated. The demodulator output signal is deinterleaved and decoded. The decoded bits are fed into an information sink.

In an optical communication system through the turbulent atmosphere, the intensity $P(u, t)$ of the received optical signal

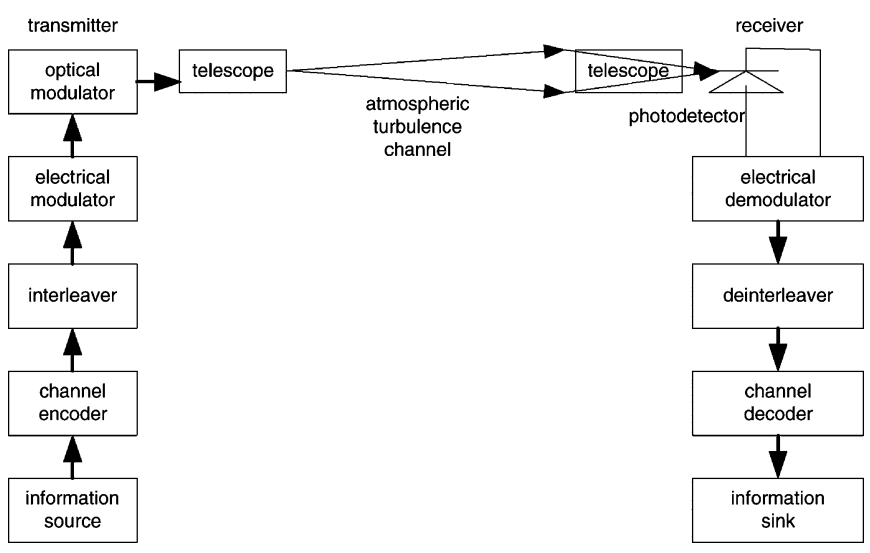

Fig. 1. Block diagram of an optical communication system through atmospheric turbulence channels.

can be written as

$$
P(u, t)=A(u, t) P_{s}(t)
$$

where $A(u, t)$ is a stationary random process for the signal scintillation caused by the atmospheric turbulence, $u$ is an event in the sample space, and $P_{s}(t)$ is the received optical intensity in the absence of turbulence. Let $R_{A}(\tau)$ be the autocorrelation function of $A(u, t)$. Assume that $R_{A}(0)$ is normalized to unity so that the average power of the converted electrical signal is normalized for analysis. When $R_{A}(0)$ is normalized, the probability distribution of a sample at any time instant is determined by one parameter defined as the scintillation level $\sigma$. The power spectral density was measured in [25] for near-ground horizontal turbulent paths of 1 and $2.5 \mathrm{~km}$ using an unmodulated laser beam. The bandwidth of the measured power spectral density was less than $1 \mathrm{kHz}$.

The random process $A(u, t)$ in (1) is well known as a log-normal process [14]-[16]. Let $x(u, t)$ be a stationary Gaussian random process with the autocorrelation function $R_{x}(\tau), R_{x}(0)=\sigma^{2}$. Define

$$
A(u, t)=k e^{x(u, t)}, \quad k>0
$$

which is a log-normal random process. The sample $A\left(u, t=t_{0}\right)$ at any time instant $t=t_{0}$ is a random variable and has the probability density function (pdf)

$$
\begin{aligned}
f(A) & =\frac{1}{\sqrt{2 \pi} \sigma A} \exp \left\{-\frac{\left(\log A-m^{\prime}\right)^{2}}{2 \sigma^{2}}\right\}, \\
m^{\prime} & =\log k, A \geq 0 .
\end{aligned}
$$

The $n$th moment of $\mathrm{A}$ is $E\left\{A^{n}\right\}=k^{n} e^{n^{2} \sigma^{2} / 2}$. To compare the BER performance, especially when coding is employed, the second moment of $A$ is normalized at the photodetector output so that the power of the electrical signal is unity. By normalizing $E\left\{A^{2}\right\}$, one has $k=\exp \left\{-\sigma^{2}\right\}$. The pdf of the random variable $A\left(u, t=t_{0}\right)$ can be written as

$$
f(A)=\frac{1}{\sqrt{2 \pi} \sigma A} \exp \left\{-\frac{\left(\log A+\sigma^{2}\right)^{2}}{2 \sigma^{2}}\right\}
$$

and

$$
E\{A\}=\exp \left\{\frac{-\sigma^{2}}{2}\right\}
$$




\section{OPTICAL COMMUNICATION EMPLOYING OOK INTENSITY MODULATION}

OOK is the dominant modulation scheme employed in existing optical communication systems through the atmosphere for commercial applications. It is well known that atmospheric turbulence causes severe performance degradation to such systems [13]. This section identifies the root cause for the performance degradation.

In an OOK system, the information data sequence is converted into an electrical signal

$$
z(u, t)=\sum_{i=-\infty}^{\infty} d_{i} g\left(t-i T_{s}\right)
$$

where $d_{i} \in\{1,-1\}$ is the signal level for the $i$ th data symbol, $g(t)$ is the shaping pulse, and $T_{s}$ is the symbol time. This electrical signal drives a laser. The intensity of the transmitted laser beam can be written as

$$
s(t)=1+\sum_{i=-\infty}^{\infty} d_{i} g\left(t-i T_{s}\right) .
$$

The received intensity of the optical beam can be written as

$$
P(t)=\frac{P}{2} A(u, t)+\sum_{i=-\infty}^{\infty} \frac{P}{2} A(u, t) d_{i} g\left(t-i T_{s}\right)
$$

where $P$ is the maximum received intensity when there is no turbulence. Hence, the received electrical signal is

$$
r(t)=K\left\{A(u, t)+\sum_{i=-\infty}^{\infty} A(u, t) d_{i} g\left(t-i T_{s}\right)\right\}+n(t)
$$

where $K$ is a constant determined by the received optical intensity and the photoelectric conversion efficiency, $n(t)$ is additive white Gaussian noise (AWGN) [9], [13], [20], [21], [26], [27]. Without loss of generality, the received electrical signal can be written as

$$
r(t)=A(u, t)+\sum_{i=-\infty}^{\infty} A(u, t) d_{i} g\left(t-i T_{s}\right)+n(t) .
$$

When there is no turbulence, i.e., $A(u, t)=1$, the received electrical signal is

$$
r(t)=1+\sum_{i=-\infty}^{\infty} d_{i} g\left(t-i T_{s}\right)+n(t) .
$$

The dc term can be filtered out without impairing the spectrum of the information signal. The BER of the optical communication system employing OOK modulation can be written as

$$
P_{e}=Q\left(\sqrt{\frac{E_{b}}{\sigma_{g}^{2}}}\right)
$$

where $E_{b}$ is the energy per bit contained in the second term in (11), and $\sigma_{g}^{2}$ is the power of the additive Gaussian noise [28]. The signal-to-noise power ratio is $\nu=E_{b} / N_{0}$ with $\sigma_{g}^{2}=N_{0} / 2$.

When atmospheric turbulence is present, the received signal is disturbed by the turbulence if the logic " 1 " is sent and the laser is turned on. In this case, the received signal can be written as

$$
\left.r(t)\right|_{s_{1}}=2 A(u, t)+n(t) .
$$

The conditional pdfs of the electrical signal at the photodetector output can be written as

$$
\begin{aligned}
& p\left(r \mid s_{0}\right)=\frac{1}{\sqrt{2 \pi} \sigma_{g}} \exp \left\{-\frac{r^{2}}{2 \sigma_{g}^{2}}\right\} \\
& p\left(r \mid s_{1}\right)=\frac{\exp \left\{-\sigma^{2} / 2\right\}}{2 \pi \sigma \sigma_{g}} \int_{0}^{\infty} \frac{1}{x^{2}} \exp \left\{-\left[\frac{\ln ^{2} x}{2 \sigma^{2}}+\frac{(r-x)^{2}}{2 \sigma_{g}^{2}}\right]\right\} d x
\end{aligned}
$$

where $s_{0}$ indicates that the logic " 0 " is sent, $s_{1}$ means that the logic " 1 " is sent, and $\sigma$ is the scintillation level. Equation (15) is obtained through a convolution of the log-normal density function and the Gaussian density function. These two conditional pdfs are asymmetric. To minimize the error probability, the receiver must have accurate knowledge of both the turbulence level and the additive noise to choose an optimum threshold, which is impossible in system implementation, pointed out in [7] and verified in the practice of free-space laser communications.

Let the detection threshold be $T>0$. The BER of an OOK system in the presence of atmospheric turbulence can be written as

$$
P_{e}(T)=p_{0} P\left(r>T \mid s_{0}\right)+\left(1-p_{0}\right) P\left(r<T \mid s_{1}\right)
$$

where $p_{0}$ is the a priori probability of sending the information bit " 0 ". Substituting (14) and (15) into (16), the BER of an OOK system can be written as

$$
\begin{aligned}
P_{e}(T)= & p_{0} Q\left(\frac{T}{\sigma_{g}}\right)+\frac{1-p_{0}}{\sqrt{2 \pi} \sigma} \exp \left\{-\frac{\sigma^{2}}{2}\right\} \\
& \times \int_{0}^{\infty} \frac{1}{x^{2}} \exp \left\{-\frac{\ln ^{2} x}{2 \sigma^{2}}\right\} Q\left(\frac{x-T}{\sigma_{g}}\right) d x
\end{aligned}
$$

where $Q(x)=\int_{x}^{\infty}(1 / \sqrt{2 \pi}) e^{-\left(t^{2} / 2\right)} d t$.

For high SNR $\nu$, one has

$$
\lim _{\nu \rightarrow \infty} P\left(r<T \mid s_{1}\right)=\lim _{\nu \rightarrow \infty} \int_{-\infty}^{T} f(x) * f_{g}(x) d x
$$

and

$$
\lim _{\nu \rightarrow \infty} P\left(r>T \mid s_{0}\right)=\lim _{\nu \rightarrow \infty} \int_{T}^{\infty} f_{g}(x) d x=0
$$

where $f(x)$ is the pdf of the scintillation random variable and $f_{g}(x)$ is the pdf of the additive noise. At high SNR, the BER is determined by both the scintillation level $\sigma$ and the threshold. The BER limit can be written as

$$
\lim _{\nu \rightarrow \infty} P_{e}(T)=\left(1-p_{0}\right) Q\left(\frac{\ln 2-\ln T}{\sigma}-\sigma\right) .
$$

For optical communication systems employing OOK through the atmospheric turbulence channel and direct demodulation with a fixed threshold, the BER cannot be infinitely small even when the SNR is very high. This explains the BER floors for OOK systems employing fixed-threshold detection, as observed 
TABLE I

BER LIMIT FOR OPTICAL COMMUNICATION SYSTEMS EMPLOYING FIXED-THRESHOLD OOK WITH THE THRESHOLD $T=e^{-\sigma^{2} / 2}$ AND ATMOSPHERIC TURBULENCE LEVEL $\sigma$

\begin{tabular}{|c|c|c|c|c|c|}
\hline$\sigma$ & 0.10 & 0.15 & 0.20 & 0.25 & 0.30 \\
\hline BER Limit & $1.4809 \mathrm{E}-12$ & $1.3670 \mathrm{E}-6$ & $1.9085 \mathrm{E}-4$ & $2.8 \mathrm{E}-3$ & $1.04 \mathrm{E}-2$ \\
\hline
\end{tabular}

in [7]. There is a BER limit as a function of both the scintillation level $\sigma$ and the threshold $T$. A low threshold may result in poor BER performance at low SNR, but better performance at high SNR. Let the threshold be the mean of the log-normal scintillation process, i.e., $T=e^{-\sigma^{2} / 2}$, by (5). The BER limit for different scintillation levels is shown in Table I. A BER limit also exists for coded optical communication systems employing fixed-threshold OOK. This is verified in Section V.

Optical communication systems employing fixed-threshold OOK intensity modulation cannot overcome the atmospheric turbulence [7]. This can be explained in the frequency domain. Following (10), the power spectral density function of the received electrical signal can be written as

$$
\mathcal{R}(f)=\mathcal{A}(f)+\mathcal{A}(f) * \mathcal{Z}(f)+\mathcal{N}(f)
$$

where $\mathcal{A}(f)$ is the power spectral density function of the turbulence process $A(u, t), \mathcal{Z}(f)$ is the power spectral density function of the transmitted signal $z(u, t), \mathcal{A}(f) * \mathcal{Z}(f)$ is the convolution, and $\mathcal{N}(f)$ is the power spectral density function of noise process. The response of the atmospheric turbulence channel to the nonzero dc term in the transmitted signal is the baseband random process $A(u, t)$ in (10), or the first term in (21). The response of the atmospheric turbulence channel to the information carrying signal $z(u, t)$ in (6) is the scintillated signal as the second term in (10) or in (21). The scintillation process $A(u, t)$ and the signal $z(u, t)$ are independent baseband random processes. Because the information carrying $\mathcal{A}(f) * \mathcal{Z}(f)$ and the noninformation $\mathcal{A}(f)$ are both in baseband, it is too difficult for a receiver to differentiate these two random processes. In other words, the demodulation is always disturbed by the first term in either (10) or (21). Therefore, the scintillation process $A(u, t)$ can severely limit demodulation performance.

\section{OPTICAL COMMUNICATION EMPLOYING SUBCARRIER PSK INTENSITY MODULATION}

The performance of optical communications system in the presence of atmospheric turbulence can be improved by separating the information carrying signal from the noninformation carrying first term in (10) or (21). The separation should be completed before the demodulator input. As long as intensity modulation is used, the first term in (10) or (21) always exists. In practice, intensity modulation is preferred for simplicity. One method is to shift in the frequency domain the information carrying second term in (10) or (21) away from the baseband random process $A(u, t)$ [23]. This section analyzes the performance of optical communication systems employing subcarrier PSK intensity modulation through the turbulent atmosphere.
Consider the optical communication system employing subcarrier PSK intensity modulation in Fig. 1. In the electrical modulator, the data sequence is modulated using PSK, which can be implemented with existing microchips at very low cost. The PSK signal is upconverted to an intermediate frequency (IF) $f_{c}$. Because the bandwidth of the scintillation process $A(u, t)$ is only a few kilohertz [25], $f_{c}$ can be chosen high enough to guarantee that the first term and the second term in either (10) or (21) do not overlap in the frequency domain. With the current RF technology, $f_{c}$ can be large enough to support several gigabits per second. Unless required by a very high data rate, $f_{c}$ should not be too large so that the terminal cost can be low. The upconverted PSK signal modulates the intensity of the laser in the transmitter. The transmitted optical intensity can be written as

$$
s(t)=1+\alpha\left[s_{i}(t) \cos \omega_{c} t-s_{q}(t) \sin \omega_{c} t\right]
$$

where $s_{i}(t)=\sum_{j} g\left(t-j T_{s}\right) \cos \phi_{j}$ is the in-phase signal and $s_{q}(t)=\sum_{j} g\left(t-j T_{s}\right) \sin \phi_{j}$ is the quadrature signal, $0<\alpha \leq 1, \omega_{c}=2 \pi f_{c}, \phi_{j}$ is the $j$ th phase symbol, $g(t)$ is the shaping pulse, and $T_{s}$ is the symbol time. The amplitude satisfies $\sqrt{s_{i}^{2}(t)+s_{q}^{2}(t)} \leq 1$ to avoid nonlinearity. Directly modulated laser diodes such as the JDS Uniphase CTR915 Series can be employed for high efficiency $\alpha=1$ and low cost. The dc component in the transmitted signal (22) is greater than zero, which is always true when intensity modulation is employed.

The received optical intensity can be written as

$$
P(t)=I_{0} A(u, t)\left\{1+\alpha\left[s_{i}(t) \cos \omega_{c} t-s_{q}(t) \sin \omega_{c} t\right]\right\}
$$

where $I_{0}$ is constant. The electrical signal at the photodetector output can be written as

$$
\begin{aligned}
I(t)= & A(u, t)+\alpha A(u, t)\left[s_{i}(t) \cos \omega_{c} t-s_{q}(t) \sin \omega_{c} t\right] \\
& +n_{i}(t) \cos \omega_{c} t-n_{q}(t) \sin \omega_{c} t
\end{aligned}
$$

where $n_{i}(t)$ and $n_{q}(t)$ are AWGN processes each with variance $\sigma_{g}^{2}$. This system is required to transmit the same optical power as that in a comparable system employing OOK in Section III.

The power spectral density of the received signal is

$$
\begin{aligned}
& \mathcal{I}(f)=\mathcal{A}(f)+\frac{\mathcal{B}\left(f-f_{c}\right)+\mathcal{B}\left(f+f_{c}\right)}{2} \\
&+\frac{\mathcal{N}\left(f-f_{c}\right)+\mathcal{N}\left(f+f_{c}\right)}{2}
\end{aligned}
$$

where $\mathcal{B}(f)=\mathcal{A}(f) * \mathcal{Z}(f)$. Assume that

$$
f_{c}>B_{A}+B_{B}
$$

where $f_{c}$ is the IF, $B_{A}$ is the single-sided bandwidth of $\mathcal{A}(f)$, and $B_{B}$ is the single-sided bandwidth of $\mathcal{B}(f)$. The first term in (24) can be filtered out by a bandpass filter, which gives the output

$$
\begin{aligned}
I_{1}(t)= & \alpha A(u, t)\left[s_{i}(t) \cos \omega_{c} t-s_{q}(t) \sin \omega_{c} t\right] \\
& +n_{i}(t) \cos \omega_{c} t-n_{q}(t) \sin \omega_{c} t .
\end{aligned}
$$


Downconverting this signal to the baseband gives the in-phase signal at the demodulator input as

$$
r_{i}(t)=\alpha A(u, t) s_{i}(t)+n_{i}(t)
$$

and the quadrature signal

$$
r_{q}(t)=\alpha A(u, t) s_{q}(t)+n_{q}(t) .
$$

Carrier phase recovery is required to obtain (28) and (29). OOK systems do not need carrier phase recovery.

For optical communication systems employing BPSK through the atmospheric turbulence channel, the BER is

$$
P_{e}=\frac{\exp \left\{-\sigma^{2} / 2\right\}}{2 \pi \sigma \sigma_{g}} \int_{0}^{\infty} x^{-2} \exp \left\{-\frac{\ln ^{2} x}{2 \sigma^{2}}\right\} Q\left(\frac{\alpha x}{\sigma_{g}}\right) d x
$$

One has

$$
\left.\lim _{\nu \rightarrow \infty} P_{e}\right|_{T=0}=Q(\infty)=0 .
$$

For optical communication systems employing QPSK, the BER can be written as

$$
P_{e}=\frac{\exp \left\{-\sigma^{2} / 2\right\}}{2 \sqrt{2} \pi \sigma \sigma_{g}} \int_{0}^{\infty} x^{-2} \exp \left\{-\frac{\ln ^{2} x}{2 \sigma^{2}}\right\} Q\left(\frac{\alpha x}{\sqrt{2} \sigma_{g}}\right) d x .
$$

When $M$-ary PSK with $M>4$ is employed, define the received signal vector $\mathbf{r}=\left[r_{i} r_{q}\right]$. Let $V=\sqrt{r_{i}^{2}+r_{q}^{2}}$ and $\Theta=$ $\arctan r_{q} / r_{i}$. The joint pdf can be written as [28]

$$
p(V, \Theta)=\frac{V}{2 \pi \sigma_{g}^{2}} \exp \left\{-\frac{V^{2}-2 r V \cos \Theta+r^{2}}{2 \sigma_{g}^{2}}\right\}
$$

where $r$ is the envelope of the received signal when the AWGN is not present. This yields the joint pdf of $V, \Theta$, and $r$ as

$$
\begin{aligned}
p(V, \Theta, r)= & \frac{V}{(2 \pi)^{3 / 2} \sigma_{g}^{2} \sigma} \\
& \times \exp \left\{-\frac{V^{2}-2 r V \cos \Theta+r^{2}}{2 \sigma_{g}^{2}}-\frac{\left(\ln r+\sigma^{2}\right)^{2}}{2 \sigma^{2}}\right\} .
\end{aligned}
$$

The symbol error rate is

$$
\begin{aligned}
P_{e}= & 1-\int_{-\pi / M}^{\pi / M} \int_{0}^{\infty} \frac{1}{2 \pi \sigma} \exp \left\{-\frac{r^{2}}{2 \sigma_{g}^{2}}-\frac{\left(\ln r+\sigma^{2}\right)^{2}}{2 \sigma^{2}}\right\} \\
& \left\{\frac{1}{\sqrt{2 \pi}}+\frac{r \cos \theta}{\sigma_{g}}\left[1-Q\left(\frac{r \cos \theta}{\sigma_{g}}\right)\right]\right. \\
& \left.\times \exp \left\{\frac{r^{2} \cos ^{2} \theta}{2 \sigma_{g}^{2}}\right\}\right\} d r d \theta .
\end{aligned}
$$

In (32) and (35), the symbol energy is normalized to unity.

Fig. 2 shows the simulated BER for optical communication systems employing subcarrier BPSK intensity modulation or OOK through atmospheric turbulence. The threshold for OOK demodulation is chosen as the mean of the random process, i.e., $e^{-\sigma^{2} / 2}$. At the scintillation level $\sigma=0.1$, an optical communication system employing subcarrier BPSK needs

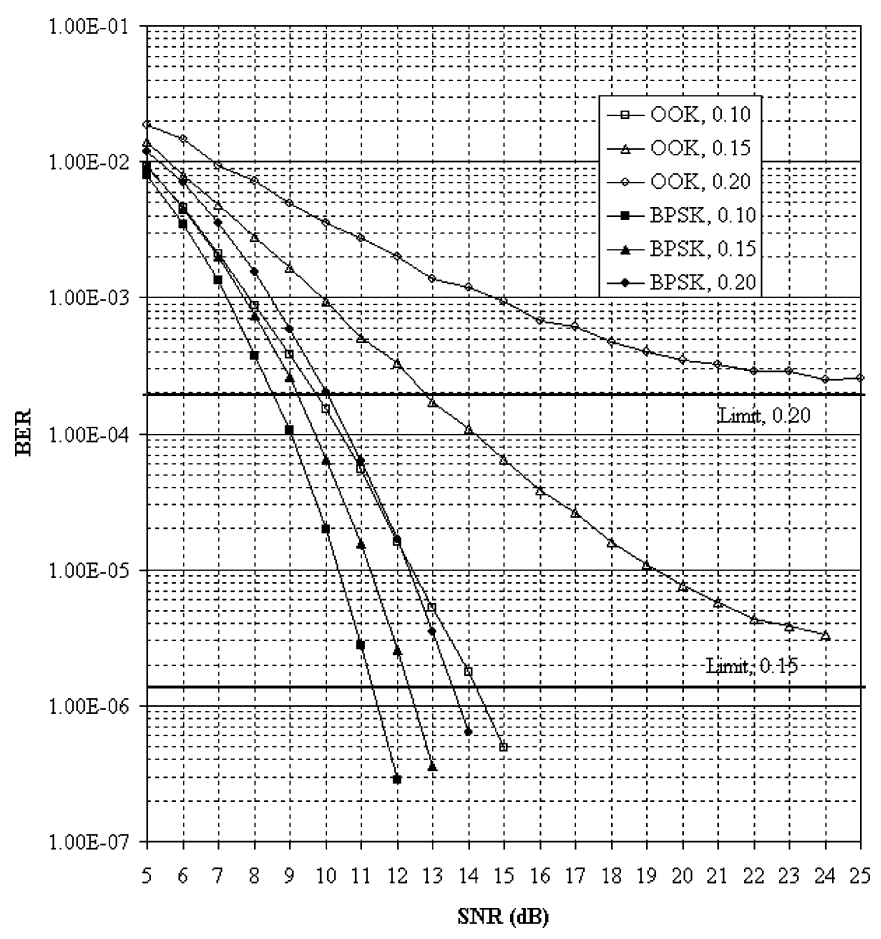

Fig. 2. BER performance of optical communication systems employing OOK or subcarrier BPSK with $\sigma \in\{0.1,0.15,0.2\}$.

$\mathrm{SNR}=11.5 \mathrm{~dB}$ to reach $\mathrm{BER}=10^{-6}$ in the presence of atmospheric turbulence, while a comparable system using OOK requires $\mathrm{SNR}=14.5 \mathrm{~dB}$ for the same BER. At the scintillation level $\sigma=0.15$, the SNR for the subcarrier BPSK system with $\mathrm{BER}=10^{-6}$ is $12.5 \mathrm{~dB}$, while the BER of an OOK system cannot reach $10^{-6}$. At $\sigma=0.2$, the subcarrier BPSK intensitymodulated system can achieve BER $=10^{-6}$ at SNR less than $14 \mathrm{~dB}$, while the BER of a comparable OOK system is never less than $10^{-4}$.

When subcarrier PSK intensity modulation is employed for optical communication, the response of the atmospheric turbulence to the nonzero dc component in the transmitted optical signal is the first term in the received signal of (24), which is a strong baseband random process of narrow bandwidth. This term is filtered out by a bandpass filter before demodulation, and does not affect the demodulator. In other words, the demodulator uses (28) and (29) to perform demodulation. For subcarrier BPSK intensity modulation, the demodulator needs to estimate the received phase in $\{0, \pi\}$, and the equivalent demodulation threshold is zero. When $M$-ary PSK with $M \geq 4$ is employed, demodulation is in the phase domain, where the demodulator needs to estimate the phase among $M$ possibilities.

It was claimed in $[22, \mathrm{Sec} . \mathrm{I}]$ that:

"In the presence of scintillation, the subcarrier PSK modulation is more suitable than OOK because of the former's ' 0 ' threshold decision."

One can think in this way for subcarrier BPSK intensity modulation, which was the focus in [22]. For subcarrier $M$-ary PSK intensity modulation with $M \geq 4$, this claim is wrong. In general, it is the filtered-out dc response before demodulation that 
allows subcarrier PSK intensity modulation to have superior demodulation performance. For $M$-ary PSK with $M \geq 4$, there does not exist any "“0” threshold decision” [23], [28].

\section{Performance of Convolutional Codes}

Optical wireless systems are severely limited by power and error correcting coding is desired. This section analyzes the performance of convolutional codes in optical communications employing subcarrier PSK intensity modulation in the presence of atmospheric turbulence.

A performance upper bound for convolutional codes was derived for memoryless channels in [29]. Let $T(D, N)$ be the generating function of all paths merging with the all-zero path in a convolutional code. The upper bound can be written as

$$
P_{B}<\sum_{i} C_{i} P_{i}
$$

where $C_{i}$ is determined by $\{d T(D, N)\} /\left.d N\right|_{N=1}=\sum_{i} C_{i} D^{i}$ and $P_{i}$ is the probability that the $i$ th bit is in error. A general performance upper bound was achieved as [29]

$$
P_{B}<\left.\frac{d T(D, N)}{d N}\right|_{N=1, D=D_{0}}=\sum_{i} C_{i} D_{0}^{i}
$$

where

$$
D_{0}=\sum_{r_{i} \in \mathbf{R}} \sqrt{P\left(r_{i} \mid-1\right) P(r \mid 1)}
$$

and $\mathbf{R}$ is the space for the received signal and $P\left(r_{i} \mid x\right)$ is the conditional probability distribution of the received signal when $x \in\{-1,1\}$ is sent.

The atmospheric turbulence is relatively slow [25] compared with the data rates in optical wireless systems. This means the scintillation is almost the same over successive bits. In other words, the channel has memory. Originally invented for the AWGN channel, decoding algorithms for convolutional codes cannot exploit the channel memory and are not optimum for atmospheric turbulence channels. Having low complexity, convolutional codes and the Viterbi decoding algorithm are efficient to correct random errors.

When the instantaneous scintillation is severe, it can cause burst errors and severely degrade decoder performance. Interleaving can be employed to change the bit sequence in coded information blocks. Although there might be successive bit errors in the received bit stream, a deinterleaver changes the bit sequence of the received information block back, and makes the occurrences of errors random [30].

Block interleaving is assumed to improve the performance of the Viterbi decoder in the presence of atmospheric turbulence [24]. The interleaving is supposed to make distributions of any pair of received sequential symbols independent. A little strong for a channel with long memory, this assumption is necessary to find the performance bound for convolutionally coded systems. When the memory effect of the channel is removed by interleaving, the Viterbi algorithm can be employed for decoding and the bound in (37) can be applied.
For optical communication system employing OOK modulation, when the turbulence is present, the conditional probability functions are asymmetric as in (14) and (15). We identify the difficulty in decoding a convolutionally coded OOK signal and try to improve it. Define a code word set $\left\{C_{0}, C_{1}, C_{2}, C_{3}\right\}$ as $C_{0}=$ $(0,0,0), C_{1}=(0,0,1), C_{2}=(0,1,0)$, and $C_{3}=(0,1,1)$. Let $P_{i j}$ be the probability that when $C_{i}$ is sent, the received signal is decoded as $C_{j}$. Although the Hamming distance between the code word $C_{2}$ and the code word $C_{1}$ is the same as the Hamming distance between the code word $C_{0}$ and the code word $C_{3}$, $P_{03} \neq P_{12}$, when atmospheric turbulence is present. In addition, one has $P_{03} \neq P_{30}$ in the presence of atmospheric turbulence. This situation becomes more obvious when the atmospheric turbulence is strong and the receiver noise power is low. When the SNR is extremely high, the error probability for transmitting " 0 " is almost zero, while the error probability for transmitting " 1 " is determined by (20). The asymmetric characteristics for OOK signals increase the complexity of the decoding algorithm. The performance analysis for coded OOK modulation will involve all paths and is very complicated.

For optical communication systems employing subcarrier PSK intensity modulation, the atmospheric turbulence affects the dc term and the information component independently in the frequency domain. The conditional pdf $p(r \mid x)$ of the received signal can be written as

$$
\begin{aligned}
& p(r \mid x)= \\
& \begin{cases}\frac{1}{2 \pi \sigma \sigma_{g}} \int_{0}^{\infty} t^{-1} \exp \left\{-\left(\frac{\left(\ln t+\sigma^{2}\right)^{2}}{2 \sigma^{2}}+\frac{(r-t)^{2}}{2 \sigma_{g}^{2}}\right)\right\} d t, & x=1 \\
\frac{1}{2 \pi \sigma \sigma_{g}} \int_{0}^{\infty} t^{-1} \exp \left\{-\left(\frac{\left(\ln t+\sigma^{2}\right)^{2}}{2 \sigma^{2}}+\frac{(r+t)^{2}}{2 \sigma_{g}^{2}}\right)\right\} d t, & x=-1\end{cases}
\end{aligned}
$$

where $x=1$ for logic " 1 " and $x=-1$ for logic " 0 ". The conditional pdfs are symmetric. By employing interleaving, the distributions of successive bits are independent. In this case, one has $P_{03}=P_{12}$ and $P_{03}=P_{30}$.

To calculate the performance upper bound for convolutional codes, the pairwise error probability $P_{k}\left(\mathbf{x}, \mathbf{x}^{\prime}\right)$ for an incorrect path $\mathbf{x}^{\prime}$ that differs in $k$ symbols from the correct path $\mathbf{x}$ is defined as

$$
P_{k}\left(\mathbf{x}, \mathbf{x}^{\prime}\right)=\sum_{\mathbf{r} \in \mathbf{R}_{\mathbf{k}}} \prod_{i=1}^{k} P\left(r_{i} \mid x_{i}\right)
$$

where $\mathbf{R}_{k}$ is the set of all vectors $\mathbf{r}=\left\{r_{1}, r_{2}, \ldots, r_{k}\right\}$ for which

$$
\prod_{i=1}^{k} \frac{P\left(r_{i} \mid x_{i}^{\prime}\right)}{P\left(r_{i} \mid x_{i}\right)}>1
$$

and $i$ runs over the $k$ code symbols in which the paths differ. Then

$$
\begin{aligned}
P_{k}\left(\mathbf{x}, \mathbf{x}^{\prime}\right) & <\sum_{\mathbf{r} \in \mathbf{R}_{k}} \prod_{i=1}^{k} P\left(r_{i} \mid x_{i}\right)\left[\frac{P\left(r_{i} \mid x_{i}^{\prime}\right)}{P\left(r_{i} \mid x_{i}\right)}\right]^{1 / 2} \\
& =\sum_{\mathbf{r} \in \mathbf{R}_{k}} \prod_{i=1}^{k}\left[P\left(r_{i} \mid x_{i}\right) P\left(r_{i} \mid x_{i}^{\prime}\right)\right]^{1 / 2} .
\end{aligned}
$$


In (39), the conditional probability function is symmetric, i.e.,

$$
p(r \mid+1)=p(-r \mid-1) .
$$

This means the set in which $\prod_{i=1}^{k}\left\{P\left(r_{i} \mid x_{i}^{\prime}\right)\right\} /\left\{P\left(r_{i} \mid x_{i}\right)\right\}>1$ is half of the space $\mathbf{R}$. Let $\mathbf{R}_{k}^{c}$ be the complement of $\mathbf{R}_{k}$. By the symmetry property, one has

$$
\begin{aligned}
& \sum_{\mathbf{r} \in \mathbf{R}_{k}} \prod_{i=1}^{k}\left[P\left(r_{i} \mid x_{i}\right) P\left(r_{i} \mid x_{i}^{\prime}\right)\right]^{1 / 2} \\
&=\sum_{\mathbf{r} \in \mathbf{R}_{k}^{c}} \prod_{i=1}^{k}\left[P\left(r_{i} \mid x_{i}\right) P\left(r_{i} \mid x_{i}^{\prime}\right)\right]^{1 / 2} .
\end{aligned}
$$

Hence, the integral can be written as

$$
\begin{aligned}
\sum_{\mathbf{r} \in \mathbf{R}_{k}} \prod_{i=1}^{k}\left[P\left(r_{i} \mid x_{i}\right)\right. & \left.P\left(r_{i} \mid x_{i}^{\prime}\right)\right]^{1 / 2} \\
= & \frac{1}{2} \sum_{\mathbf{r} \in \mathbf{R}} \prod_{i=1}^{k}\left[P\left(r_{i} \mid x_{i}\right) P\left(r_{i} \mid x_{i}^{\prime}\right)\right]^{1 / 2} .
\end{aligned}
$$

With the symmetry of the conditional pdf, an upper bound is achieved as

$$
P_{B}<\left.\frac{1}{2} \frac{d T(D, N)}{d N}\right|_{N=1, D=D_{0}}
$$

This upper bound is tighter than that in (37). The approach is applicable to any symmetric signal constellation. For continuous channels, $D_{0}$ can be written as

$$
\begin{aligned}
D_{0} & =\sum_{r \in R} \lim _{\Delta r \rightarrow 0} \sqrt{p(r \mid-1) \Delta r p(r \mid 1) \Delta r} \\
& =\int_{-\infty}^{\infty} \sqrt{p(r \mid-1) p(r \mid 1)} d r .
\end{aligned}
$$

When interleaving is employed, the bound calculation is simplified. Without memory effect, the decoder does not need to calculate the transition probabilities of the Markov process in [20], and the decoding algorithm is simplified and optimum.

Fig. 3 shows simulated BER for an optical communication system employing convolutional code and OOK modulation. The scintillation level is $\sigma \in\{0.1,0.2,0.3,0.4,0.5\}$. The convolutional code is of rate $1 / 2$, constraint length 3 , and polynomials [5 7]. At the scintillation level $\sigma=0.1$, the SNR is approximately $7.2 \mathrm{~dB}$ at BER $=10^{-6}$. The coding gain is about $7.3 \mathrm{~dB}$, much larger than that in the AWGN channel. When $\sigma \geq 0.3$, the BER curves exhibit floors. At $\sigma=0.5$, the BER is greater than $4 \times 10^{-4}$ when $\mathrm{SNR}=18 \mathrm{~dB}$.

Fig. 4 shows simulated BER for a comparable system employing subcarrier BPSK intensity modulation and the same convolutional code as in the simulated OOK system in Fig. 3. At the scintillation level $\sigma=0.1$, the coding gain is approximately $4.5 \mathrm{~dB}$ at $\mathrm{BER}=10^{-6}$. The coding gain increases to more than $5.8 \mathrm{~dB}$ at the scintillation level $\sigma=0.2$ and $\mathrm{BER}=10^{-6}$. At $\sigma=0.5$, the coded subcarrier BPSK intensity-modulated system achieves BER $=10^{-6}$ at $\mathrm{SNR}=11.75 \mathrm{~dB}$. The BER of the coded OOK system is always greater than $4 \times 10^{-4}$. Therefore, subcarrier PSK intensity modulation is superior to OOK, when

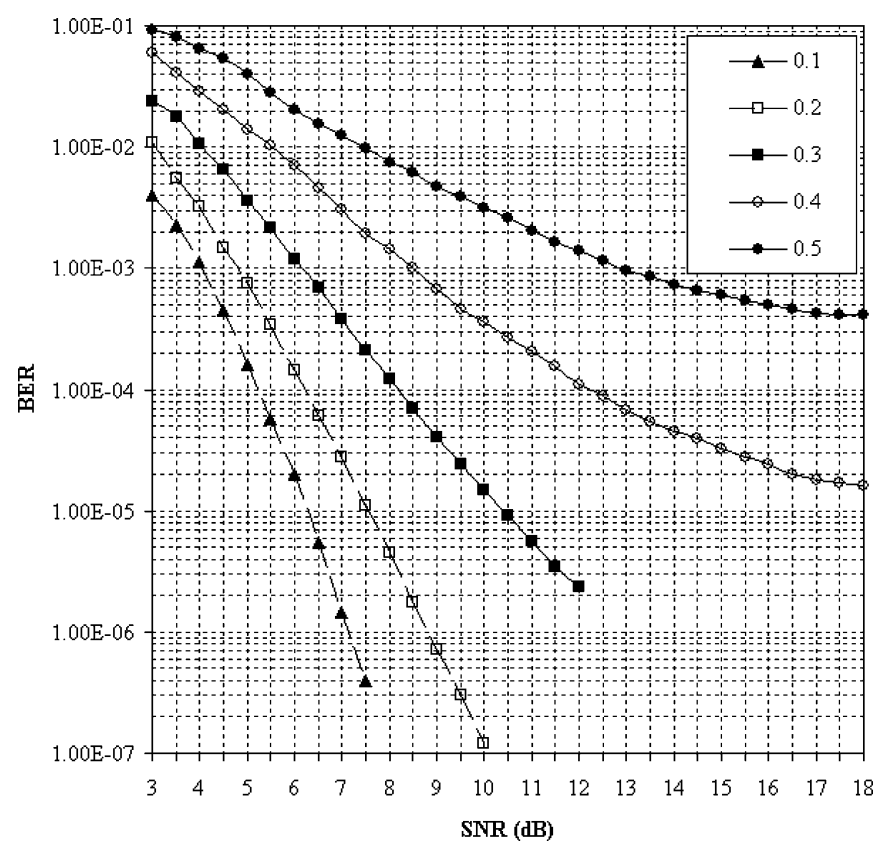

Fig. 3. BER performance of optical communication systems employing convolutional code and OOK with $\sigma \in\{0.1,0.2,0.3,0.4,0.5\}$. The convolutional code has rate $1 / 2$ and constraint length 3 .

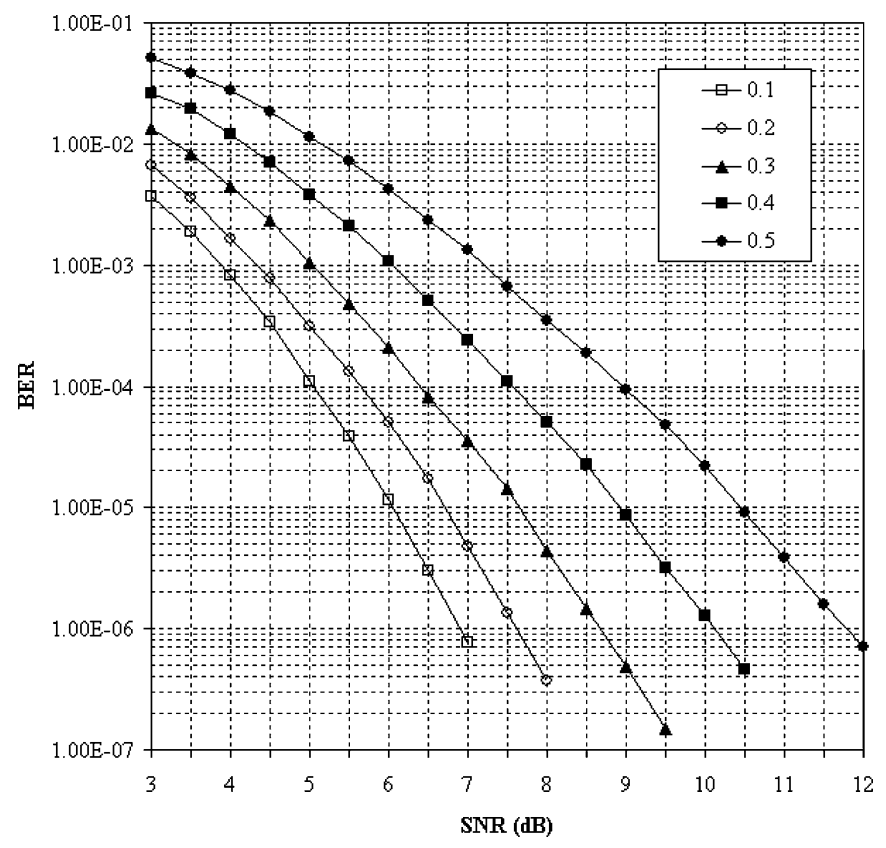

Fig. 4. BER performance of optical communication systems employing convolutional code and subcarrier BPSK with $\sigma \in\{0.1,0.2,0.3,0.4,0.5\}$. The convolutional code has rate $1 / 2$ and constraint length 3 .

convolutional codes are employed in optical communication systems through atmospheric turbulence.

Fig. 5 shows the simulated BER for optical wireless communication systems employing a rate $1 / 3$ convolutional code and subcarrier BPSK intensity modulation. Ideal interleaving is applied to the coded information block and deinterleaving is employed before decoding. The convolutional code has constraint length 3 and the polynomials [ $\left[\begin{array}{lll}4 & 5 & 7\end{array}\right]$. It can be seen 


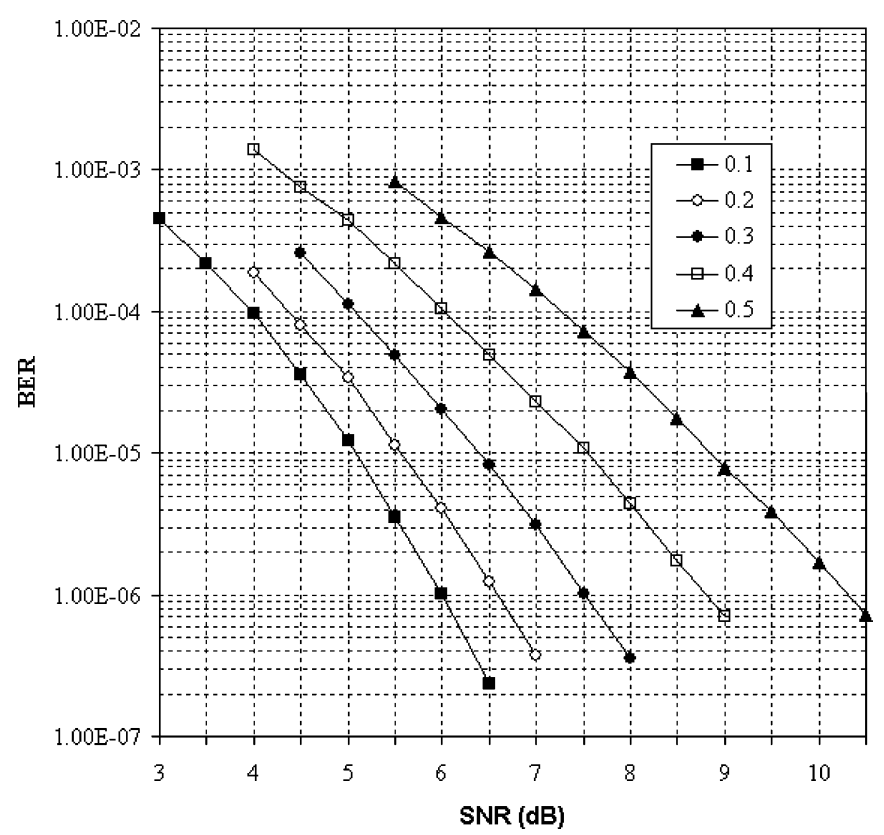

Fig. 5. BER performance of optical communication systems employing convolutional code subcarrier BPSK with $\sigma \in\{0.1,0.2,0.3,0.4,0.5\}$. The convolutional code has rate $1 / 3$ and constraint length 3 .

that at $\sigma=0.5, \mathrm{BER}=10^{-6}$ can be achieved at approximately $10.3 \mathrm{~dB}$.

When $\sigma=0.4$ and the correlation time of the scintillation is the same as the bit duration, the BER performance is almost the same as that in [21]. This is because the interleaving makes the scintillation independent between any pair of adjacent bits so that the correlation is nonzero only in the bit interval.

At $\sigma=0.4$ and BER $=10^{-6}$, simulations have shown that interleaving can help gain approximately $1.2 \mathrm{~dB}$ when the scintillation correlation is 1000 times of the bit duration. Therefore, interleaving can help improve system performance in optical communications through relatively slow scintillation channels.

Fig. 6 compares the simulation results and bounds calculated using (46) for the convolutional code with rate $1 / 2$, constraint length 3 , and polynomials [ 5 7 $]$. At BER $=10^{-6}$, the simulated BER curve is about $0.6 \mathrm{~dB}$ to the left of the calculated bound for $\sigma=0.1$, and approximately $0.9 \mathrm{~dB}$ for $\sigma=0.4$. The numerical results show the bound is tighter than that in [21]. The difference between the simulated result and the calculated bound is caused by that in some subset of $\mathbf{R}_{k}$, it is true that

$$
\prod_{i=1}^{k} \frac{p\left(r_{i} \mid x_{i}^{\prime}\right)}{p\left(r_{i} \mid x_{i}\right)} \gg 1 .
$$

As the scintillation level increases, the gap between the calculated bound and the simulated curve increases too. This is because when the scintillation level increases, to achieve the same BER, the system has to work at a higher SNR. The pdf of the received signal is dominated by the pdf of the scintillation process. The integral to approximate $P_{k}$ on the set that satisfies (48) becomes larger.

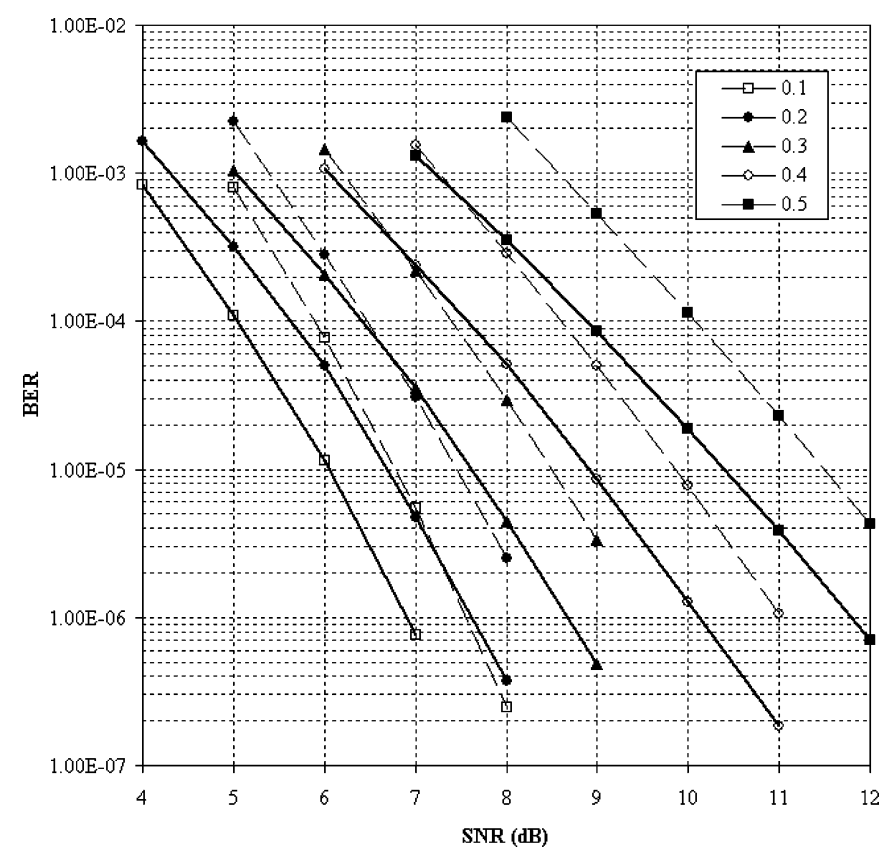

Fig. 6. BER performance bounds of optical communication systems employing convolutional code subcarrier BPSK with $\sigma \in\{0.1,0.2,0.3,0.4,0.5\}$. The convolutional code has rate $1 / 2$ and constraint length 3 . The bounds are plotted as dashed lines, while simulated results are in solid lines.

\section{CONCLUSION}

Subcarrier PSK intensity modulation can help to effectively mitigate the signal scintillation caused by atmospheric turbulence in optical communications. The BER limit for fixedthreshold OOK modulation is found. By deriving BER for both OOK and subcarrier BPSK intensity modulation, it is shown that the subcarrier PSK intensity modulation is superior to OOK intensity modulation in the presence of atmospheric turbulence.

Convolutional codes are studied for optical communication systems through the atmospheric turbulence channel. Coded subcarrier PSK intensity-modulated systems are superior to coded systems employing OOK. At the scintillation level $\sigma=0.5$, the BER of an OOK system employing a convolutional code with rate $1 / 2$ and constraint length 3 is always greater than $2 \times 10^{-4}$, while a comparable system employing subcarrier BPSK can achieve BER $=10^{-6}$ at $\mathrm{SNR}=11.75 \mathrm{~dB}$. Interleaving is used to overcome the relatively slow scintillation. Simulations have shown that interleaving can help gain $1.2 \mathrm{~dB}$ at $\sigma=0.4$ and $\mathrm{BER}=10^{-6}$, when a convolutional code with rate $1 / 3$ and constraint length 3 is used for an atmospheric channel with correlation spread of thousands of bit durations. Interleaving can help reduce decoder complexity significantly and simplify performance analysis for decoding.

An upper bound is derived for the performance of optical wireless communication systems employing convolutional coded subcarrier BPSK modulation. This bound is more than $0.5 \mathrm{~dB}$ tighter than the bound in [21] at $\mathrm{BER}=10^{-6}$ and $\sigma=0.4$. Therefore, subcarrier PSK intensity modulation and convolutional codes can be employed in optical communications through atmospheric turbulence channels to improve performance and reduce transmission power. 


\section{REFERENCES}

[1] A. Acampora, "Last mile by laser," Sci. Amer., vol. 287, pp. 48-53, Jul. 2002.

[2] P. F. Szajowski, G. Nykolak, J. J. Auborn, H. M. Presby, G. E. Tourgee, and D. Romain, "Key elements of high-speed WDM terrestrial freespace optical communications systems," Proc. SPIE, vol. 3932, pp. 2-14, 2000.

[3] D.-Y. Song, Y.-S. Hurh, J.-W. Cho, J.-H. Lim, D.-W. Lee, J.-S. Lee, and Y. Chung, " $4 \times 10$ Gbps terrestrial optical free space transmission over $1.2 \mathrm{~km}$ using an EDFA preamplifier with $100 \mathrm{GHz}$ channel spacing," Opt. Exp., vol. 7, pp. 280-284, Oct. 2000.

[4] L. C. Andrews and R. L. Phillips, Laser Beam Propagation Through Random Media. Bellingham, WA: SPIE, 1998.

[5] I. I. Kim, E. Woodbridge, V. Chan, and B. R. Strickland, "Scintillation measurements performed during the limited-visiblity lasercom experiment," Proc. SPIE, vol. 3266, pp. 209-220, 1998.

[6] G. R. Ochs, R. R. Bergman, and J. R. Snyder, "Laser-beam scintillation over horizontal paths from 5.5 to 145 kilometers," Appl. Opt., vol. 59, pp. 231-234, Feb. 1969

[7] J. H. Shapiro and R. C. Harney, "Burst-mode atmospheric optical communication," in Proc. 1980 Nat. Telecommun. Conf., pp. 27.5.1-27.5.7.

[8] R. S. Lawrence and J. W. Strohbehn, "A survey of clear air propagation effects relevant to optical communications," Proc. IEEE, vol. 58, no. 10, pp. 1523-1545, Oct. 1970.

[9] X. Zhu and J. M. Kahn, "Free-space optical communication through atmospheric turbulence channels," IEEE Trans. Commun., vol. 50, no. 8, pp. 1293-1300, Aug. 2002.

[10] D. L. Fried, "Optical heterodyne detection of an atmospherically distortion wave front," Proc. IEEE, vol. 55, no. 1, pp. 57-67, Jan. 1967.

[11] D. L. Fried, G. E. Mevers, and M. P. Keister, Jr., "Measurements of laser-beam scintillation in the atmosphere," J. Opt. Soc. Amer., vol. 59, pp. 1455-1460, Nov. 1969.

[12] J. H. Churnside, "Aperture averaging of optical scintillations in the turbulent atmosphere," Appl. Opt., vol. 30, pp. 1982-1994, May 1991.

[13] X. Zhu, J. M. Kahn, and J. Wang, "Mitigation of turbulence-induced scintillation noise in free-space optical links using temporal-domain detection techniques," IEEE Photon. Technol. Lett., vol. 15, no. 4, pp. 623-625, Apr. 2003.

[14] P. H. Deitz and N. J. Wright, "Saturation of scintillation magnitude in near-earth optical propagation," J. Opt. Soc. Amer., vol. 59, pp. 527-535, May 1969

[15] G. R. Ochs, R. R. Bergman, and J. R. Snyder, "Saturation of laser-beam scintillation under conditions of strong atmospheric turbulence," Appl. Opt., vol. 59, pp. 226-227, Feb. 1969.

[16] D. A. DeWolf, "Are strong irradiance fluctuations log normal or Rayleigh distributed," J. Opt. Soc. Amer., vol. 57, pp. 787-797, Jun. 1967.

[17] I. I. Kim, H. Hakakha, P. Adhikari, E. Korevaar, and A. Majumdar, "Scintillation reduction using multiple transmitters," Proc. SPIE, vol. 2990, pp. 102-113, 1997.

[18] S. M. Haas and J. H. Shapiro, "Capacity of wireless optical communications," IEEE J. Sel. Areas Commun., vol. 21, no. 8, pp. 1346-1357, Oct. 2003.

[19] R. K. Tyson, "Bit-error rate for free-space adaptive optics laser communications," J. Opt. Soc. Amer., vol. 19, pp. 753-758, Apr. 2002.

[20] X. Zhu and J. M. Kahn, "Markov chain model in maximum-likelihood sequence detection for free-space optical communication through atmospheric turbulence channels," IEEE Trans. Commun., vol. 51, no. 3, pp. 509-516, Mar. 2003.

[21] X. Zhu and J. M. Kahn, "Performance bounds for coded free-space optical communications through atmospheric turbulence channels," IEEE Trans. Commun., vol. 51, no. 8, pp. 1233-1239, Aug. 2003.

[22] W. Huang, J. Takayanagi, T. Sakanaka, and M. Nakagawa, "Atmospheric optical communication system using subcarrier PSK modulation," IEICE Trans. Commun., vol. E76-B, no. 9, pp. 1169-1177, Sep. 1993.

[23] Q. Lu, Q. Liu, and G. S. Mitchell, "Performance analysis for optical wireless communication systems using sub-carrier PSK intensity modulation through turbulent atmospheric channel," in Proc. IEEE Global Telecommun. Conf., Dallas, TX, Nov. 29-Dec. 3 2004, pp. 18721875.

[24] Q. Liu and Q. Lu, "Subcarrier PSK intensity modulation for optical wireless communications through turbulent atmospheric channel," in Proc. IEEE Int. Conf. Commun., Seoul, Korea, May 16-20, 2005, pp. 17611765.
[25] B. M. Levine, E. A. Martinsen, A. Wirth, A. Jankevice, M. ToledoQuinones, F. Landers, and T. L. Bruno, "Horizontal line-of-sight turbulence over near-ground paths and implications for adaptive optics corrections in laser communications," Appl. Opt., vol. 37, pp. 4553-4560, Jul. 1998.

[26] M. Srinivasan, J. Hamkins, B. Madden-Woods, A. Biswas, and J. Beebe "Laboratory characterization of silicon avalanche photodiodes (APDs) for pulse-position modulation (PPM) detection," IPN Progress Rep. 42-146, Aug. 15, 2001, pp. 1-14.

[27] M. Uysal, S. M. Navidpour, and J. Li, "Error rate performance of coded free-space optical links over strong turbulence channels," IEEE Commun. Lett., vol. 8, no. 10, pp. 635-637, Oct. 2004.

[28] J. G. Proakis, Digital Communications. New York: McGraw-Hill, 2001.

[29] A. J. Viterbi, "Convolutional codes and their performance in communication systems," IEEE Trans. Commun., vol. 19, no. 5, pp. 751-772, Oct. 1971.

[30] H. R. Sadjadpour, N. J. A. Sloane, M. Salehi, and G. Nebe, "Interleaver design for turbo codes," IEEE J. Sel. Areas Commun., vol. 19, no. 5, pp. 831-837, May 2001.

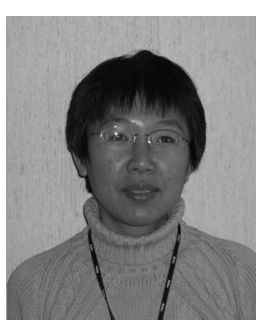

Jia Li (M'02) received the B.S. degree in electronics and information systems from Peking University, Beijing, China, in 1996, and the M.S.E. and Ph.D. degrees in electrical engineering from the University of Michigan, Ann Arbor, in 1997 and 2002, respectively.

She is currently an Assistant Professor of Engineering at Oakland University, Rochester, MI Her current research interests include ultra-wideband (UWB) sensor networks for automotive vehicles, communication theory, statistical shape modeling, image segmentation, multimodal image registration, and image-guided therapy.

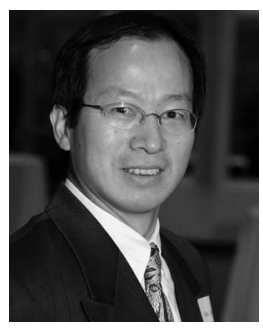

John Q. Liu (S'93-M'99-SM'06) received the B.S. degree in electronics and information systems and the M.S. degree in computer science from Peking University, Beijing, China, in 1990 and 1992, respectively, the M.S. degree in electrical engineering from New Mexico State University, Las Cruces, in 1993, and the $\mathrm{Ph} . \mathrm{D}$. degree in electrical engineering from the University of Southern California, Los Angeles, in 1996.

From 1996-2000 he was a Senior Member of Technical Staff with Hughes Network Systems. He is currently an Associate Professor at Wayne State University, Detroit, MI. His current research interests include radio network security, communications networks, ultra-wideband communications, optical wireless communications, modulation and coding, synchronization, signal design, and detection.

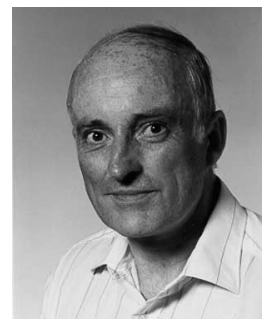

Desmond P. Taylor (M'65-SM'90-LF'07) was born in Noranda, QC, Canada, on July 5, 1941. He received the B.Sc. Eng. and M.Sc. Eng. degrees in electrical engineering from Queen's University, Kingston, ON, Canada, in 1963 and 1967, respectively, and the Ph.D. degree in electrical engineering from McMaster University, Hamilton, ON, in 1972.

From July 1972 to June 1992, he was with the Communications Research Laboratory and Department of Electrical Engineering, McMaster University. In July 1992, he joined the University of Canterbury, Christchurch, New Zealand, where he is currently the Tait Professor of Communications. His current research interests include spectrally efficient, high-performance, digital transmission systems and problems in multiple access and networking. He is the author or coauthor of more than 180 papers published in international journal. He is the holder of two U.S. patents in spread spectrum communications.

Dr. Taylor is a Fellow of the Royal Society of New Zealand, the Engineering Institute of Canada, and the Institute of Professional Engineers of New Zealand. 